\title{
Sosa, DeRose y las Creencias Seguras
}

\author{
IGNACIO ViLARÓ
}

\begin{abstract}
According to Sosa, in order for a truthful belief to count as knowledge it must be safe (a modal notion which is similar, although not identical, to that of sensitivity). An alleged advantage of this condition of safety, over that of sensitivity, has to do with its capacity to provide a Moorean response to the skeptical Cartesian. DeRose criticizes safety both from a pragmatic and a semantic point of view. I present a slightly different version of his critique putting aside some incidental issues and clarifying what is at stake in his main charge. Sosa's response is inadequate because it deals only with peripheral aspects.
\end{abstract}

Keywords: Safety; Mooreanism; skepticism; Sosa; DeRose.

\section{La seguridad de las creencias y la respuesta mooreana al Escéptico}

Los epistemólogos se ocupan recurrentemente de reflexionar sobre un argumento paradójico —el argumento escéptico a partir de la ignorancia, $A I$ :

(1) No sé que no soy un cerebro en una cubeta (sin manos), estimulado electroquímicamente para tener precisamente las experiencias que de hecho tengo

(2) Si 1 , entonces 3

(3) Con. No sé que tengo manos

Como recuerda Sosa, algunas de las opciones prominentes para enfrentar esta paradoja son las siguientes.

Escéptico: acepta 1, 2 y c

Nozick, et al.: acepta 1, no-c y no-2

Moore: acepta 2, no-c y no-1

Contextualismo: en los contextos escépticos 1, 2 y c expresan proposiciones verdaderas. En los contextos usuales, no-con expresa una proposición verdadera. ${ }^{1}$

En "How to defeat opposition to Moore" Sosa señala los problemas de la segunda y de la cuarta alternativa (1999, p.143-5). No ofrece argumentos en contra del Escepticismo, pero es claro que considera que cualquier alternativa no escéptica es, en principio, preferible. Por lo tanto, al menos si no consideramos otras opciones, Sosa puede argumentar a favor del mooreanismo criticando la segunda y la cuarta opción.

Principia 18(1): 135-151 (2014).

Published by NEL — Epistemology and Logic Research Group, Federal University of Santa Catarina (UFSC), Brazil. 
Desde luego, su argumento será más poderoso si tenemos razones para pensar que el mooreanismo no sufre de los defectos de las demás alternativas no escépticas. Sosa afirma que varios problemas de las opciones no mooreanas se deben a su asunción de que la sensibilidad es una condición necesaria para que una creencia verdadera de que p sea conocimiento. ${ }^{2}$

Una creencia de $S$ de que $p$ es sensible sii si $p$ fuera falsa, $S$ no creería que $p$. Sea ' $\phi \rightarrow \psi$ ' un condicional subjuntivo. Una creencia de $S$ de que $p, C s(p)$, es sensible sii (3) $\neg p \rightarrow \neg C s(p)$ (Sosa 1999, p.146)

El cargo principal que se suele alegar en contra de quienes niegan la segunda premisa de AI es que ello los compromete con negar alguna versión adecuada del Principio de Clausura del Conocimiento ( $P C C$ ), generalmente aceptado como esencialmente correcto. ${ }^{3}$ Sosa, junto con DeRose, apelan a una manera bastante vívida de ilustrar esta dificultad general. Quienes niegan 2 deben aceptar lo que DeRose llama conjunciones abominables, como por ejemplo, "sé que tengo manos, pero no sé que no soy un cerebro en una cubeta $(C C)$, sin manos, estimulado electroquímicamente para tener precisamente las experiencias que de hecho tengo" (Sosa 1999, p.143) (DeRose 1995, p.28). Contra Nozick, Sosa afirma que mi creencia de que no soy un CC no tiene por qué ser sensible para contar como conocimiento. El mooreano niega 1 , pero acepta $2 .^{4}$

Pero el contextualista tampoco se compromete con la verdad universal de las conjunciones abominables (ya que, por ejemplo, si estamos en un contexto escéptico, no sé que tengo manos). Y, de hecho, él tiene una explicación simple de por qué 1 puede resultarnos tan plausible: en los contextos escépticos, 1 es verdadera. A primera vista, el mooreano parece no tener una explicación disponible de este hecho. ¿O sí? Según Sosa, una virtud de sustituir la sensibilidad por la seguridad es que podemos, a la vez, adoptar el mooreanismo y explicar la plausibilidad de 1 .

Una creencia de $S$ de que $p$ es segura sii si $S$ creyera que $p, p$ sería el caso. Una creencia de $S$ de que $p, C s(p)$, es segura sii (4) $C s(p) \rightarrow p$. La sensibilidad y la seguridad no son equivalentes, porque los condicionales subjuntivos no respetan la contraposición. (Sosa 1999, p.146)

Si el conocimiento exige la seguridad de nuestras creencias, y no su sensibilidad, entonces tenemos una explicación elegante de la plausibilidad de 1: lo que sucede es que confundimos la sensibilidad con la seguridad (Sosa 1999, p.147-8). Y, si somos sensibles, deberíamos distinguirlas.

Esto dejaría en tablas al mooreano y al contextualista, pero Sosa cree que la asunción de la sensibilidad como una condición necesaria para conocer debilita la posición contextualista. De acuerdo con este último: 
[...] it is only the skeptic's position that is ever endorsable, in whatever context, inasmuch as the very endorsing of that position so changes the context as to make its endorsement correct. (Sosa 1999, p.145)

Este es un resultado que querríamos evitar porque parece amenazar la viabilidad de la disolución contextualista de la paradoja $A I$. Parece que le concedemos al Escéptico la facultad de destruir el conocimiento cuando le plazca, simplemente mencionando una alternativa escéptica. ${ }^{5}$ Personalmente, considero que hay cuando menos algo de verdad en la crítica de Sosa. No obstante, DeRose se niega explícitamente a aceptar esta lectura de su teoría. ${ }^{6}$ Sin embargo, para los fines de este trabajo, asumiremos que Sosa tiene razón en este punto. ${ }^{7}$ Detenernos a discutir sobre esta cuestión nos alejaría irremediablemente de nuestra preocupación central. Lo que queremos es evaluar algunos problemas que genera la posición de Sosa, asumiendo que esta es, cuando menos en principio, atractiva.

\section{La inadecuación pragmática de los condicionales de seguridad}

Como señala DeRose, la proferencia de un condicional subjuntivo como (4) es problemática. Dada una lectura natural del condicional, podríamos parafrasear (4) del siguiente modo: "si hubiera creído que p, entonces p habría sido el caso" (2004, p.29). Digamos que yo estoy casado y que soy feliz. En un contexto conversacional (como el presente), en el que se presuponen ambas cosas, digo (5) "si estuviera casado, sería feliz". La reacción natural ante mi proferencia es la de desconcierto. ¿Qué es lo que se supone que quiero hacer al proferir (5)? Esta situación sugiere una primera generalización: los condicionales subjuntivos presuponen la falsedad de su antecedente. Siendo más precisos, diremos que (A) un hablante $X$ puede proferir, de modo no defectuoso, un condicional subjuntivo, ' $\phi \rightarrow \psi$ ', solo si presupone que $\phi$ es falso.

Para contar con una noción más clara de presuposición, acordemos que, de aquí en más, trabajaremos con la noción pragmática stalnakeriana. La idea central es que la presuposición es una acción llevada adelante por los hablantes para comunicarse de modo eficiente. Las presuposiciones son simplemente disposiciones a asumir la verdad de ciertas proposiciones. Los hablantes asumen la verdad de ciertas proposiciones y asumen, a su vez, que los demás hablantes hacen lo propio. Desde luego, las presuposiciones no tienen por qué ser verdaderas. Sin embargo, para simplificar las cosas, asumiré de aquí en más que no hay presuposiciones falsas. Siguiendo a Stalnaker, utilizaré $\mathrm{C}$, un conjunto de mundos posibles, para representar aquello que se presupone en una conversación. $\mathrm{C}$ es aquel conjunto de mundos posibles tal que se excluyen de él las negaciones de las proposiciones presupuestas por los participantes de la conversación. Por ejemplo, en la conversación entre el lector y quien suscribe, 
ni la proposición de que no estoy casado, ni la de que soy infeliz pertenecen a C (Stalnaker 1970, 1974).

Si la generalización (A) fuera correcta, sería relativamente simple defender la taxonomía tradicional de las oraciones condicionales, según la cual:

(S) Todos los condicionales subjuntivos son contrafácticos.

(I) Todos los condicionales indicativos son no-contrafácticos. ${ }^{8}$

Sin embargo, (A) tiene contraejemplos.

6. Si Jones hubiera tomado arsénico, hubiera mostrado exactamente los mismos síntomas que de hecho muestra (Anderson 1951).

Es natural que encontremos (6) en un contexto en el cual queremos argumentar a favor de la verdad del antecedente $p$. Por lo tanto, en un contexto así, sería absurdo suponer que $p$ es falso. Por lo tanto (S) es falso. Obviamente, no se trata en este caso de un argumento deductivo. El argumento tiene la siguiente forma: si $p$ entonces $q$; $q$ es el caso; por lo tanto es probable que $p$. Es posible ofrecer un argumento deductivamente válido que muestra el mismo punto. "Si Jones hubiera tomado arsénico, estaría vomitando y con náuseas. Jones no está vomitando y con náuseas. Por lo tanto, Jones no tomó arsénico.” Obviamente, quien utiliza este argumento no puede presuponer que el antecedente del condicional es falso ya que esa es precisamente la conclusión a la que pretende arribar. Dado que es posible argumentar correctamente de este modo sin cometer una petición de principio, podemos concluir que esta defensa de la taxonomía tradicional es incorrecta.

Quizás teniendo en cuenta dificultades como esta, DeRose dice que los condicionales subjuntivos presuponen, en algún sentido, que su antecedente es falso (2004, p.29). ${ }^{9}$ Ahora bien, incluso si la defensa estándar de la taxonomía tradicional tiene dificultades, sigue siendo cierto, como señala DeRose, que la proferencia de un condicional subjuntivo, en un contexto en el que se presupone la verdad de su antecedente, es pragmáticamente defectuosa. Este fenómeno sigue demandando una explicación de nuestra parte. De hecho, podemos modificar levemente (A) para tal efecto, atendiendo a la noción stalnakeriana de presuposición. He aquí una propuesta alternativa.

(B) Un hablante $X$ puede proferir, de modo no defectuoso, un condicional subjuntivo, ' $\phi \rightarrow \psi$ ', solo si no presupone $\phi$.

La exigencia recogida en (B) es más débil que la recogida en (A). Según (A), puedo proferir correctamente un condicional subjuntivo sólo si $a \notin C$, esto es, sólo si C contiene únicamente mundos no-A. En cambio, según (B), puedo proferir correctamente un condicional subjuntivo sólo si no presupongo que $\phi$ es verdadero. Hay 
dos posibles maneras en las que puedo satisfacer este requerimiento: (i) $a \notin C$, (ii) $(a \in C)$ y $(\neg a \in C)$. Según (i) C contiene únicamente mundos no-A. Según (ii) C contiene tanto mundos-A como mundos no-A. La posibilidad excluida por (B) es que $\mathrm{C}$ contenga únicamente mundos-A. (B) predice, correctamente, que mi proferencia de (5) resultará defectuosa en un contexto, como el presente, en el que presuponemos que estoy casado. Sin embargo, no tiene el resultado inadecuado de juzgar, a priori, que el argumento de Jones es, necesariamente, una petición de principio. En ese caso, $\mathrm{C}$ contiene tanto mundos-A como mundos no-A.

¿Cuál es la importancia de estos asuntos para la noción de seguridad? Esta peculiaridad pragmática de los condicionales subjuntivos parece amenazar la relevancia de la seguridad para aislar la clase de creencias conocidas. Dada la peculiaridad pragmática mencionada, no tenemos intuiciones pre-teóricas muy sólidas acerca del valor de verdad de estos condicionales; por ello, parecería que la condición de Sosa es inútil para la tarea que se propone. Veamos por qué. La manera de juzgar la adecuación intuitiva de la seguridad, como una condición epistémica para saber que $p$, supone considerar casos en los que, intuitivamente, sabemos que $p$ y evaluar si, en esos casos, nuestras creencias son seguras. Obviamente, Sosa admite:

(7) Si $X$ sabe que $p$, entonces se satisface (4)

La lectura pretendida de (7) es aquella según la cual " $X$ sabe que $p$ " recibe una interpretación intuitiva, no una redefinición técnica determinada. (7) afirma simplemente que, para ser conocida, una creencia debe ser segura.

Pero, obviamente, como nosotros, Sosa admite:

(8) Si $X$ sabe que $p$, entonces $X$ cree que $p$.

Ahora bien. Hagamos el ejercicio de evaluar la corrección de (7). Para ello, supongamos que $X$ sabe que $p$. Si (7) es verdadera, se satisface (4), esto es, si $X$ hubiera creído que $p, p$ hubiera sido el caso. Pero en el presente contexto conversacional, hemos supuesto que $X$ sabe que $p$ y, dado (8), en este contexto, presuponemos que $X$ cree que $p \cdot{ }^{10}$ Por lo tanto, dado (B), en este contexto, el condicional de seguridad resulta pragmáticamente defectuoso. Por lo tanto, parece que no podemos evaluar si es o no es cierto que la seguridad es una condición necesaria para que una creencia de que $p$ sea conocida. Dado el modo en el que funciona nuestro lenguaje, simplemente no podemos decidir si Sosa ha propuesto una condición epistémica razonable.

No sucede lo mismo con (3). En general, pensamos que lo siguiente es verdad:

(9) Si $X$ sabe que $p$, entonces es verdad que $p$.

(10) Si es verdad que $p$, entonces $p$.

Principia 18(1): 135-151 (2014). 
Si queremos evaluar la corrección de la sensibilidad como una condición necesaria para saber que $p$, nos encontraremos en un contexto conversacional en el que presuponemos que $p$. Tomemos un caso paradigmático de una creencia que nos parece conocer: que tenemos manos. Supongamos que $X$ sabe que tiene manos. En el contexto en el que hacemos esa suposición, presupondremos que es verdad que $X$ tiene manos. Pero es perfectamente razonable preguntarnos qué hubiera pasado si $X$ no tuviera manos, tal como nos exige la condición de sensibilidad.

Es cuando menos misterioso preguntarnos qué hubiera pasado si A hubiera sido el caso, mientras presuponemos, a la vez, que A es el caso. Esta inadecuación pragmática es problemática para Sosa porque, al parecer, nos impide preguntarnos significativamente si, en efecto, una condición necesaria para saber que p, es que mi creencia de que $p$ sea segura. El siguiente pasaje de DeRose expresa esta incredulidad:

[...] I will be quite unable to check the safety account of knowledge against examples to determine if it is right. For such a task will consist of taking examples where $S$ has a true belief that $p$, and then asking of them whether it is also true that if $S$ had believed that $p, p$ would have been true, and then checking those results against intuitions about whether $S$ knows that $p$ in the cases. But it's hopeless, at least for me, to try to carry out this process. Wherever $S$ does believe that $p$, and $p$ is true, the conditional If $S$ had believed that $p, p$ would have been the case will just strike me as weird and wrong; there's just no way that I'll be able to get stable and discriminating reactions that might match up with intuitions about whether the subject knows in the various cases: "Well, here the [true/true subjunctive] conditional seems true, but here it seems false." Apparently I'm not alone, given the typical attitude taken toward true/true subjunctives by students of conditionals. Thus, I find accounts of knowledge like Sosa's [...] that put so much weight on true/true subjunctives highly problematic. ${ }^{11}$ (DeRose 2004, p.31)

\section{La ausencia de un modelo semántico apropiado}

Quizás sea cierto que la proferencia de un condicional subjuntivo cuyo antecedente se presupone verdadero genera un absurdo pragmático. No obstante, Sosa podría decir que siempre que las cosas funcionen bien semánticamente, esta es una preocupación menor. Si la semántica formal es clara, y si las discriminaciones entre condicionales verdaderos y falsos de la forma (4) se alinean con nuestras reacciones epistémicas pre-teóricas, entonces no hay grandes problemas para el análisis.

Lamentablemente para Sosa, la semántica estándar para los condicionales subjuntivos no es útil para sus propósitos. Y las modificaciones obvias a dicha semántica, que se podrían idear favoreciendo su análisis, invalidan el Modus Ponens (u otros principios intuitivamente válidos). Sosa nos debe un modelo semántico que haga lo que su análisis requiere. Debe ofrecer una alternativa no estándar para la semántica

Principia 18(1): 135-151 (2014). 
de los condicionales subjuntivos. Pero este es un precio muy elevado. Hasta que no veamos cómo se supone que funcionaría dicha semántica alternativa, el análisis de Sosa queda en entredicho.

Como señala DeRose, según el análisis estándar, todos los condicionales subjuntivos que tienen un antecedente y un consecuente verdaderos, son verdaderos (2004, p.29). Como dijimos, presumiblemente Sosa acepta (8) (9) y (10). Ahora bien, ¿cómo debemos juzgar la adecuación extensional del análisis de Sosa? Debemos preguntarnos si (7) es verdadero; esto es, debemos preguntarnos si, efectivamente, siempre que juzgamos intuitivamente que $X$ sabe que p, se satisface (4). Pero, según la semántica estándar, (4) se satisface siempre que su antecedente y su consecuente sean verdaderos. El problema para Sosa es que, en todos los casos epistémicamente relevantes, (4) posee un antecedente y un consecuente verdaderos. ¿Por qué? Porque Sosa acepta (8) (9) y (10). Por lo tanto, la tarea de juzgar si todo caso en el que intuitivamente $X$ sabe que p es un caso en el que $X$ tiene una creencia segura de que $\mathrm{p}$, se transforma en la tarea de juzgar si las creencias verdaderas de que p satisfacen (4). Pero, según la semántica estándar, todos esos condicionales son verdaderos. Por lo tanto, la teoría de Sosa tiene como resultado que toda creencia verdadera constituye conocimiento (DeRose 2004, p.30).

Desde luego, Sosa debe utilizar una semántica alternativa para sus propósitos epistemológicos. En particular, debe utilizar una semántica en la que algunos condicionales subjuntivos que tienen antecedente y consecuente verdaderos resulten verdaderos, y en la que algunos resulten falsos. ${ }^{12}$ Para terminar esta sección, recordaré por qué no parece posible modificar conservadoramente la semántica estándar para dichos propósitos. Sosa debe ofrecer una semántica heterodoxa.

Debemos la teoría semántica estándar a Lewis y a Stalnaker (Lewis 1973; Stalnaker 1968). Sus teorías difieren en algunos puntos de detalle, pero no en lo que nos concierne a nosotros ahora. Veamos por que, de acuerdo con la semántica de Stalnaker, cualquier condicional subjuntivo cuyos dos componentes son verdaderos es verdadero. Tomemos como ejemplo el condicional (5) "si estuviera casado, sería feliz". Segun Stalnaker, un condicional subjuntivo, ' $\phi \rightarrow \psi$ ', es verdadero, en un mundo base $\alpha$, sii $\phi$ es verdadero en $f s(\phi, \alpha) .{ }^{13} f s$ es una función de selección, que toma la proposición antecedente $\phi$ (equivalente a un conjunto de mundos posibles) y algun mundo base $\alpha$, en el que se evalua el condicional, y arroja un mundo posible $\beta$. Intuitivamente, $\beta$ es el mundo posible mas cercano al mundo base en el que vale el antecedente del condicional. Una de las condiciones semánticas que pone Stalnaker para $f s$ es que para todos los antecedentes $\phi$, y mundos base $\alpha$, si $\phi$ es verdadero en $\alpha$, entonces $f s(\phi, \alpha)=\alpha$. Sea $\alpha=$ el mundo actual. Dada esta semántica, 5 sera verdadera sii soy feliz y falsa sii soy infeliz. Consiguientemente, 5 es verdadera. En general, cualquier condicional subjuntivo cuyos componentes son ambos verdaderos es verdadero.

Principia 18(1): 135-151 (2014). 
Si Sosa quisiera modificar la semántica de Stalnaker, debería rechazar la restricción semántica anterior sobre $f s$. Pero si lo hace, invalidaría el Modus Ponens. Sea @ el mundo actual. Supongamos ahora que estoy casado, pero que no soy feliz. En estas condiciones profiero 5. Supongamos, ademas, que $f s(\phi, \alpha)=w_{1}$, con $w_{1} \neq @$, y que $w_{1}$ es un mundo- $\psi$, en nuestro ejemplo, un mundo en el que soy feliz. En este caso, (5) es verdadera, es verdad que estoy casado, pero es falso que yo sea feliz. La restricción semántica de Stalnaker no solo es intuitiva: es necesaria para validar el Modus Ponens. ${ }^{14}$

\section{4. ¿Son relevantes las proferencias para la Epistemología?}

La posición de Sosa tiene una dificultad innegable. Se coloca demasiado peso sobre un condicional subjuntivo que, presuponemos, tiene un antecedente verdadero. En general, estos condicionales sufren de un defecto pragmático sistemático. Por ello, el análisis corre el riesgo de no poder levantar vuelo. Y, como vimos, la semántica tradicional de los contrafácticos no puede acudir en la ayuda de Sosa en este punto. Todos los condicionales contrafácticos con ambos componentes verdaderos son verdaderos en la semántica estándar. No sólo eso: no parece haber una manera adecuada de modificar dicha semántica de modo de evitar este problema.

Pero quizás Sosa no necesite modificar la semántica tradicional. Es cierto, él debe evitar el absurdo pragmático mencionado. Pero quizás, para tal efecto, sea suficiente con señalar la completa irrelevancia de las proferencias, de las presuposiciones de los hablantes y del contexto conversacional para hacer Epistemología. ${ }^{15}$ Después de todo, lo que necesitamos es determinar si una condición necesaria para que $S$ sepa que $p$ es que tenga una creencia segura de que $p$, esto es, una creencia que satisfaga el condicional 4: "si $S$ hubiera creído que $p, p$ sería verdadero". Pero uno puede pensar que para determinar cuál es el valor de verdad de 4 no es necesario apelar a las presuposiciones de los participantes de la discusión epistemológica, ni a sus proferencias, ni a ningún contexto conversacional específico. Si, en efecto, todo ello resulta innecesario, entonces señalar la extrañeza pragmática mencionada es ortogonal al asunto epistemológicamente importante. Y uno puede pensar que, efectivamente, todo ello resulta innecesario porque cuando discutimos sobre Epistemología nos preocupamos (o deberíamos preocuparnos) por caracterizar el conocimiento, más allá de cualquier acto de habla particular que podamos llevar adelante. Según esta manera de ver las cosas, la mera existencia de una extrañeza pragmática como la señalada nos debe tener sin cuidado.

Sin embargo, esta objeción resulta apresurada. No olvidemos que es el propio Sosa quien caracteriza la fuerza apelando a un condicional contrafáctico. Pero las teorías lógico-lingüísticas tradicionales sobre estos condicionales reconocen desde el 
vamos su sensibilidad contextual. Por lo tanto, apelar a las presuposiciones contextuales, a las proferencias y a los contextos de diálogo resulta, muchas veces, inevitable.

Cuando hacemos pragmática nos ocupamos de los usos específicos de ciertas expresiones en ciertos contextos. Cuando hacemos semántica nos ocupamos de las propiedades de ciertos tipos de expresiones, dejando entre paréntesis los usos concretos en las diversas situaciones de habla. Por ejemplo, describimos cuales deben ser las condiciones semánticas (sin importar los usos particulares) que debe cumplir una función para ser una función de selección de la semántica stalnakeriana. O discutimos cuales deben ser las condiciones que debe cumplir una relación para ser una relación de similitud de la semántica de Lewis; para ello, no tenemos por qué atender a la pragmática. Afortunadamente, esta caracterización semántica de las condiciones de verdad de los contrafácticos resulta suficiente para caracterizar sus propiedades lógicas. Para asegurarnos de que este es el caso basta con mirar los sistemas formales desarrollados por Lewis o Stalnaker y comprobar que, más allá de divergencias de detalle, logran caracterizar adecuadamente las inferencias pre-teóricas que involucran condicionales contrafácticos.

Pero ni Lewis ni Stalnaker suponen que, de este modo, sea posible otorgar valores de verdad a todos, ni siquiera a la mayoría, de los condicionales contrafácticos del lenguaje natural. Simplemente, las condiciones semánticas sobre las relaciones de similitud no determinan de modo suficiente la esfera de mundos pertinentes para realizar la evaluación - como explica Lewis (1973, p.91-5); tampoco las condiciones semánticas sobre la función de selección determinan de modo suficiente cuál es el mundo posible en el que debemos evaluar si vale o no vale el consecuente de un contrafáctico, en la teoría de Stalnaker. ${ }^{16}$ En este caso, la semántica opera con un parámetro que debe ser llenado contextualmente. ${ }^{17}$ Lewis es muy claro al respecto:

Overall similarity consists of innumerable similarities and differences in innumerable respects of comparison, balanced against each other according to the relative importances we attach to those respects of comparison. Insofar as these relative importances differ from one person to another, or differ from one occasion to another, or are indeterminate even for a single person on a single occasion, so far is comparative similarity indeterminate.(1973, p.91)

All this is not so special to the comparative similarity of worlds that appears in my analysis of counterfactuals. It is the same sort of vagueness that arises if I say that Seattle resembles San Francisco more closely than it resembles Los Angeles. Does it? That depends on whether we attach more importance to the surrounding landscapes, the architecture, the dominant industries, the political temper, the state of the arts, the climate, the public transportation system, the form of the city government, or what. [...] any problem posed by my use of comparative similarity differ only in degree, not in kind, from problems about similarity that we would be stuck with no matter what

Principia 18(1): 135-151 (2014). 
we did about counterfactuals. Somehow, we do have a familiar notion of comparative overall similarity [...] However mysterious that notion may be, if we can analyze counterfactuals by means of it we will be left with one mystery in place of two. (1973, p.92)

The truth conditions for counterfactuals are fixed only within rough limits; like the relative importances of respect of comparison that underlie the comparative similarity of worlds, they are a highly volatile matter, varying with every shift of context and interest. (1973, p.92)

Nuestro objetor cree que es equivocado mencionar los aspectos pragmáticos cuando hacemos Epistemología. Sin embargo, la semántica clásica de los contrafácticos reconoce su sensibilidad contextual. Es simplemente falso que podamos, en general, prescindir de la pragmática para evaluar los valores de verdad de los contrafácticos. ${ }^{18} \mathrm{Y}$ es claro que no podemos hacerlo en el caso que nos ocupa. En Epistemología se suele asumir que si $S$ sabe que $p$, es verdad que $p$ y $S$ cree que $p$. En el contexto de conversación pertinente esta es una presuposición natural e ineludible.

\section{La respuesta de Sosa a DeRose}

Las secciones 2 y 3 resumen los que considero son los aspectos más importantes, contenidos en la crítica de DeRose a la noción de seguridad epistémica. La respuesta que ofrece Sosa a estos cargos es insuficiente porque atiende únicamente a ciertos asuntos periféricos, involucrados de modo accidental con los cargos principales. Quizás esta respuesta está motivada por el texto de DeRose. Quizás no lo está, y se trata simplemente de un recurso tendencioso de Sosa, que lee el texto como más le conviene. ${ }^{19}$ En cualquier caso, lo que es claro es que la respuesta no atiende los asuntos centrales de la crítica, tal y como fueron presentados aquí.

Según Sosa, DeRose lanza dos cargos principales en contra de su posición (además de otros cargos generales que no nos conciernen): (1) que los condicionales de seguridad no hacen el trabajo que él les asigna y (2) que, en cualquier caso, la seguridad y la sensibilidad no se contraponen (Greco 2004, p.278). Es probable que Sosa esté pensando en los pasajes que van desde la página 31 a la 33, sin contar la sección 7, del artículo de DeRose (2004). En primer lugar, allí se le da mucho peso a la rareza pragmática de los condicionales de seguridad. Esos pasajes sugieren que, al final del día, DeRose apuesta que la incorrección del análisis de seguridad deriva exclusivamente de dicha dificultad. ${ }^{20}$ La crítica involucrada aquí es, básicamente, la contenida en la segunda sección de este trabajo. En segundo lugar, DeRose desafía las paráfrasis intuitivas alternativas del condicional de seguridad, favorecidas por Sosa. No sólo porque le parecen forzadas, sino porque le parece dudoso que 
se contrapongan a la sensibilidad, como requeriría la explicación de la plausibilidad intuitiva de la primera premisa escéptica. Según DeRose, la contrapositiva de la sensibilidad es S1 "Si $X$ hubiera creído que $p, p$ hubiera sido el caso" y no alguna de las lecturas preferidas por Sosa S2-S4 (2004, p.32-3). ${ }^{21}$

Estos pasajes justifican (parcialmente) la interpretación que hace Sosa de la crítica de DeRose. Y la respuesta que se le da a estos cargos es quizás discutible pero, esencialmente, correcta. Sosa señala que DeRose no puede prohibirle que él estipule el significado del condicional de fuerza como más le plazca (Greco 2004, p.279). Y si resulta que, bajo su estipulación preferida, la sensibilidad se contrapone a la fuerza (dada la lectura adecuada), y que no surge la rareza pragmática que amenaza al análisis desde el inicio, entonces el análisis no tiene ninguno de los problemas de los que se lo acusaba. ${ }^{22}$

El problema es que esta es una respuesta insuficiente al problema real involucrado en la crítica de DeRose. Es difícil ver de que modo podríamos obtener una mejor comprensión de la corrección/incorrección del análisis, discutiendo acerca cuál es la paráfrasis intuitiva adecuada del condicional de seguridad. Incluso concediéndole a Sosa su derecho de leer el condicional subjuntivo como le plazca, desde un punto de vista intuitivo, las propiedades semánticas de ' $\phi \rightarrow \psi$ ' no se alteraran un ápice. Si existiera una semántica adecuada para los propósitos de Sosa, entonces podríamos pasar a discutir acerca de la extrañeza pragmática involucrada en proferir un condicional de seguridad. Pero es dudoso que haya una semántica asi.

\section{Conclusión}

La rareza pragmática que surge al proferir un condicional subjuntivo con ambos componentes verdaderos amenaza al análisis desde el inicio porque, al parecer, éste no puede levantar vuelo. No tenemos el tipo de intuiciones discriminadoras claras que necesitaríamos para saber si la seguridad es una condición necesaria para saber que $p$. Quizás esta dificultad se podría superar si la semántica formal del condicional subjuntivo nos diera lo que pedimos. La tarea sería determinar si siempre que tenemos la intuición preteórica de que $X$ sabe que $p, X$ posee una creencia verdadera $\mathrm{y}$ segura de que $p$ (donde el veredicto sobre la seguridad lo da la semántica formal). Si el condicional de seguridad es verdadero en todos y sólo los casos en los que tenemos la intuición de que sabemos que $p$ (y falso en todos y sólo los casos en los que tenemos la intuición de que no sabemos que $p$ ), entonces el análisis es prometedor. Pero la semántica estándar no nos provee un remedio para nuestra perplejidad pragmática. El resultado inmediato, y desastroso, que se seguiría de adoptarla, es que toda creencia verdadera contará como conocimiento. Sosa nos debe una semántica formal alternativa. Hasta que no tengamos esa semántica, es inútil discutir acerca de posibles paráfrasis alternativas del condicional de seguridad.

Principia 18(1): 135-151 (2014). 
Ante esta situación hay dos alternativas obvias que puede adoptar el simpatizante de Sosa. En primer lugar, puede intentar ofrecer una semántica alternativa a la clásica que no genere el resultado indeseado y que preserve la validez del Modus Ponens. Tal tarea queda pendiente para quien pretenda restaurar la postura mooreana oficial de Sosa. No obstante, todavía no tenemos disponible dicha semántica y no tenemos buenas razones para pensar que la tendremos en algún momento. ${ }^{23}$ En segundo lugar, puede definir la seguridad de las creencias sin apelar esencialmente a un condicional contrafáctico (como lo hace por ejemplo Pritchard (2008) para quien $S$ tiene una creencia segura de que $p$ cuando tiene una creencia que resulta verdadera en la mayoría de los mundos posibles cercanos en los que $S$ cree que $p$ ). Esta salida tiene la ventaja de ahorrarnos la difícil (quizás imposible) tarea de ofrecer una semántica no estándar para los condicionales contrafácticos.

Sin embargo, al renunciar de este modo a definir la seguridad apelando a un condicional contrafáctico, se renuncia al mismo tiempo a la teoría del error oficial propuesta por Sosa. Recordemos que, según el mooreano, la primera premisa de $A I$ es falsa. Pero entonces: ¿por qué nos parece verdadera? Como recuerda DeRose (1995), la parte difícil de la paradoja escéptica (a decir verdad, la parte difícil de cualquier paradoja) es explicar por qué una premisa que nos pareció verdadera era realmente falsa. Sosa había propuesto una elegante teoría del error: confundimos un condicional contrafáctico con otro, confundimos la sensibilidad con la fuerza. Pensamos, erróneamente, que los condicionales contrafácticos se contraponen. Pero no lo hacen (Sosa 1999, p.147-8). Sin embargo, una vez que renunciamos a definir la seguridad apelando a un condicional contrafáctico renunciamos, por ello mismo, a esta explicación. Por lo tanto, cae la versión de Sosa de una respuesta mooreana a la paradoja cartesiana.

Quizás por ello, en A Virtue Epistemology (Sosa 2007), Sosa vuelve a recurrir a los condicionales contrafácticos y a nuestra supuesta confusión de la sensibilidad con la fuerza, como una manera de explicar por qué la primera premisa de la paradoja escéptica nos parece intuitivamente verdadera, pese a ser realmente falsa.

What is required for the safety of a belief is that not easily would it fail by being false, or untrue. A belief that $p$ is safe provided it would have been held only if (most likely) p. [...] By contrast, someone's belief that $p$ is sensitive if and only if were it not so that $p$, he would not (likely) believe that $p$.

It is $[\ldots]$ quite plausible to think that such conditionals contrapose, as do material conditionals; plausible, but still incorrect. [...] Accordingly, a belief can be safe without being sensitive. Radical skeptical scenarios provide examples. Take one's belief that one is not a brain in a vat fooled by misleading sensory evidence into so believing. That belief is safe without being sensitive. We can thus defend Moorean commonsense by highlighting the

Principia 18(1): 135-151 (2014). 
skeptic's confusion of safety with sensitivity. Although our belief that we are not radically fooled is not sensitive, it is still safe, since not easily would that belief be false. Radical scenarios are ones that not easily would materialize. (Sosa 2007, p.25)

No obstante, pese a la insistencia de Sosa, hasta que no veamos cuál es la supuesta semántica no estándar de los condicionales contrafácticos que evita el resultado indeseable de que toda creencia verdadera resulte conocida (a la vez que valida el Modus Ponens, y otras inferencias intuitivamente válidas), este movimiento no está justificado. O bien se nos ofrece la semántica requerida, o bien se apela a una nueva teoría del error, que no recaiga esencialmente en nuestra supuesta confusión entre dos condicionales contrafácticos que no se contraponen. En cualquier caso, esta laguna en la Epistemología de Sosa amenaza con viciar irreparablemente su solución a la paradoja escéptica cartesiana.

\section{Acknowledgments}

Agradezco los comentarios de dos jueces anónimos que me permitieron mejorar este trabajo.

\section{Referencias}

Anderson, A. R. 1951. A Note on Subjunctive and Counterfactual Conditionals. Analysis 11: 35-8.

Cogburn, J.; Jeffrey, W. R. 2013. Safety and the True-True Problem. Pacific Philosophical Quarterly 93(4): 246-67.

DeRose, K. 1995. Solving the Skeptical Problem. The Philosophical Review 104(1): 1-52.

- 2004. Sosa, safety, sensitivity, and skeptical hypotheses. In: J. Greco (2004), 22-41.

- 2010. Insensitivity is back, baby! Philosophical Perspectives 24(1): 161-87.

Greco, J. 2004. Ernest Sosa and his critics. Malden, MA: Blackwell Pub.

Hawthorne, J. 2005. The Case for Closure. In: Contemporary Debates in Epistemology. Blackwell, 26-42.

Lewis, D. K. 1973. Counterfactuals. Cambridge: Harvard University Press.

McGlynn, A. 2012. The problem of true-true counterfactuals. Analysis 72(2): 276-85.

Pritchard, D. 2008. Sensitivity, Safety, and Anti-Luck Epistemology. In: J. Greco (ed.) The Oxford Handbook of Skepticism, 437-55. New York: Oxford University Press.

Sosa, E. 1999. How to Defeat Opposition to Moore. Nous 33(s13): 141-53. . 2007. A virtue epistemology. New York: Oxford University Press.

Stalnaker, R. 1968. A Theory of Conditionals. In: Studies in Logical Theory, 98-112. Oxford: Blackwell.

- 1970. Pragmatics. Synthese 22(1-2): 272-89.

- 1974. Pragmatic Presuppositions. In: R. Stalnaker (1999), 47-62.

Principia 18(1): 135-151 (2014). 
1999. Context and content: essays on intentionality in speech and thought. New York: Oxford University Press.

Stanley, J. G. S. Z. 2000. On Quantifier Domain Restriction. Mind and Language 15(2): 219261.

Vilaró, I. 2014. Un desafío al Contextualismo Epistémico. Tópicos, Revista de Filosofía 46: 9-50.

Von Fintel, K. 1997. The Pressupposition of Subjunctive Conditionals. MIT Working Papers in Linguistics: 29-44.

Walters, L. (forthcoming). Possible World Semantics and True-True Counterfactuals. Pacific Philosophical Quarterly. Disponible en: http://static.squarespace.com/static/513f9c0de4 b040273acd061c/t/51c18e4ae4b01e46e9399f43/1371639370094/Possible \%20Worlds \%20Semantics \%20and \%20True-True \%20Counterfactuals.pdf

IGNACIO VILARÓ

UNAM

ignaciovilaro@gmail.com

Resumo. De acordo com Sosa, para que uma crença verídica conte como conhecimento ela deve ser segura (uma noção modal similar, mas não idêntica, à de sensibilidade). Uma alegada vantagem desta condição de segurança sobre aquela de sensibilidade está relacionada com a sua capacidade de prover uma resposta Mooreana ao cético cartesiano. DeRose critica a segurança de tanto de um ponto de vista pragmático quanto semântico. Apresento uma versão levemente diferente de sua crítica, deixando de lado alguns pontos incidentais e clarificando o que está em jogo em sua principal acusação. A resposta de Sosa é inadequada pois trata apenas de aspectos periféricos.

Palavras-chave: Segurança; mooreanismo; ceticismo; Sosa; DeRose.

\section{Notas}

${ }^{1}$ Si bien hay muchas maneras de implementar esta idea general contextualista, para los fines de este trabajo asumiré la versión más elaborada del Contextualismo hasta el momento, en lo que concierne al problema escéptico. Tal versión es la que ofrece DeRose (1995). Sosa hace lo propio (1999).

2 "What follows will explore sensitivity-based opposition to plain Moorean common sense. We shall find that several of the most striking attacks on plainness rest essentially, in one way or other, on some assumed requirement of sensitivity. Replacing sensitivity with safety would in one stroke undercut all such attacks." (Sosa 1999, p.143)

${ }^{3}$ Un PCC refinado es, por ejemplo, el que ofrece Hawthorne "If one knows P and competently deduces $\mathrm{Q}$ from $\mathrm{P}$, thereby coming to believe $\mathrm{Q}$, while retaining one's knowledge that P, one comes to know that Q." (2005, p.29).

${ }^{4}$ Aunque Sosa admite que él podría tener problemas con el PCC (1999, p.149), en este trabajo dejaremos esa preocupación de lado.

Principia 18(1): 135-151 (2014). 
${ }^{5}$ En relación a la superioridad del Mooreanismo sobre el Contextualismo, a la hora de evitar el Escepticismo, dice Sosa (nota 14) "I mean that we in our reflection and in our discussions in journal and seminar, avoid skepticism; we can say right here and now that we do know various things, and not just that we say "I know" correctly in various contexts not now our own." (1999, p.152)

${ }^{6}$ Ver, por ejemplo, la nota 10 de DeRose (2010).

${ }^{7}$ En Vilaró (2014) muestro que el Contextualismo Epistémico tiene dificultades sistemáticas para implementar su estrategia anti-escéptica. En particular, muestro que las maneras más razonables de interpretar la teoría de DeRose, y que no suponen una mala interpretación de su obra (como reclama DeRose en la nota 10 de DeRose 2010), nos llevan a callejones sin salida.

${ }^{8}$ Cf. Von Fintel.

${ }^{9}$ En otro momento, refiriéndose nuevamente a la proferencia de un condicional subjuntivo, en un contexto en el que se presupone la verdad de su antecedente dice: "The conditional seems to presuppose that Tom isn't tall..." (2004, p.29). Cursivas agregadas.

${ }^{10}$ Esta derivación asume la corrección del siguiente principio: si presuponemos A, y presuponemos que A implica lógicamente $\mathrm{B}$, presuponemos B. Más allá de la posible aparición de ciertos problemas de omnisciencia presuposicional, parece que este principio resultaría adecuado, realizando los ajustes del caso. La discusión que podría suscitarse aquí es similar a la discusión sobre la versión apropiada de PCC. Por ello uno esperaría una actitud similar de Sosa respecto de la adecuación del principio, grosso modo. Para el caso que nos ocupa, presuponer "si A entonces B" supone eliminar de C todos los mundos A y no-B. Presuponer A supone eliminar de C todos los mundos no-A. Queda claro que, en ese caso, C no tendrá ningún mundo no-B. Otra asunción de este razonamiento, aparentemente no problemática, es que, si, en $\mathrm{t} 1$, un hablante introduce abiertamente el supuesto de que $p$, en $\mathrm{C}$, y no hay resistencia respecto de su acción, su audiencia, luego de t1, presupone que $p$ en $\mathrm{C}$.

${ }^{11}$ DeRose parecería hablar aquí de un proceso de evaluación que supone (erróneamente) que Sosa propone la seguridad como una condición suficiente para que una creencia verdadera de que p resulte conocida. Si leemos literalmente el pasaje, la tarea consistiría en evaluar si una creencia verdadera y segura de que $p$ resulta intuitivamente conocida. En realidad, Sosa se compromete únicamente con la necesidad de su condición modal. No obstante, el problema señalado por DeRose es real (y es, de hecho, el mismo que ya señalé más atrás). La rareza pragmática del condicional de seguridad, en el contexto de conversación relevante, nos impide tener las intuiciones claras y discriminatorias que precisaríamos para emprender la tarea de análisis. La dificultad es, en esencia, que el análisis no puede siquiera comenzar. Seguramente, este pasaje no encierra ningún error de DeRose, cuando mucho un apresuramiento al exponer una idea correcta. En otros pasajes, en los que se ocupa de las dificultades semánticas, expone con precisión el punto central (DeRose 2004, p.30).

12 Claro está, el simpatizante de la seguridad deberá encontrar una semántica no ad-hoc que sea, cuando menos, igual de explicativa que la semántica estándar. Sosa necesita una semántica con esas características para poder evaluar la corrección/incorrección de su análisis. Desde luego, necesita, además, que la semántica de los resultados adecuados (DeRose 2004, p.30).

${ }^{13}$ Stalnaker ofrece una teoría unificada de los condicionales indicativos y subjuntivos. Pero este detalle no es importante para nosotros.

Principia 18(1): 135-151 (2014). 
${ }^{14}$ Surgen problemas análogos si pretendemos modificar la semántica de Lewis en el sentido requerido por Sosa. Según la semántica de Lewis, un condicional contrafáctico (subjuntivo) es no vacuamente verdadero sii entre los mundos- $\phi$, algunos mundos- $\psi$ están más cerca de @ que cualquier mundo-no- $\psi$. Según esta variedad de la semántica estándar, si $\phi$ es verdadero, ' $\phi \rightarrow \psi$ ' es verdadero sii $\psi$ es verdadero. Sosa podría modificar la condición de verdad, agregándole al final "exceptuando al mundo actual". Esta modificación genera el mismo contraejemplo a la validez del Modus Ponens cuando es verdad, en @, que estoy casado y es falso, en @, que soy feliz. Puede suceder en ese caso que entre los mundos- $\phi$, algunos mundos- $\psi$ esten mas cerca de @ que cualquier mundo-no- $\psi$ (exceptuando a @). Esto sugiere que la reforma semántica que debe emprender el simpatizante de Sosa es profunda. Una opción para el sería usar las consideraciones de Lewis, en 1.7, sobre weak centering (1973, p.26-31). Al adoptar esa linea, el simpatizante puede conservar el Modus Ponens. Sin embargo, como se muestra en (Walters 2013) esta salida tambien genera resultados contraintuitivos. Walters considera y rechaza dos posibles modificaciones a la semántica estándar, la propuesta por Cogburn y Roland, y la propuesta por McGlynn. En ambos casos, el problema es que las modificaciones invalidan esquemas que querríamos preservar. El resultado provisorio es que la semántica estándar es la más balanceada de las opciones disponibles.

${ }^{15}$ Agradezco a un Juez anónimo por presionar este punto.

${ }^{16}$ Stalnaker también es claro al respecto. "The aim of the analysis is to give a perspicuous representation of the formal structure of conditionals - to give the form of their truthconditions. Even if nothing substantive is said about how antecedents select counterfactual possible world, the analysis still has non-trivial, and in some cases surprising, consequences for the logic of conditionals."(Stalnaker 1999, p.69) Y más adelante: "Relevant respects of similarity are determined by the context, and the semantics abstracts away from the context by taking it as an unexplained given."(1999, p.69) El lector atento habrá notado que estos pasajes proceden de "Indicative Contitionals". No obstante, sería incorrecto pensar que son por ello irrelevantes para la teoría de los contrafácticos. De hecho, Stalnaker propone una teoría semántica unificada para los contrafácticos y los indicativos.

${ }^{17}$ Las condiciones de verdad son precisas pero apelan a un parámetro contextual. Es natural pensar que sucede algo similar en otros casos. Por ejemplo, el dominio de cuantificación de los cuantificadores del lenguaje natural debe ser restringido contextualmente. Cuando te pregunto si todas las cervezas están frías no me refiero a todas las cervezas del universo. Hay un significado constante (unas condiciones de verdad) y unos valores de verdad variables. Puede haber muchas discrepancias acerca de cómo es, exactamente, que el contexto de uso restringe la interpretación de los cuantificadores. Pero existe un enorme consenso en que sucede algo de este tipo. (Stanley 2000)

${ }^{18}$ En la concepción clásica de los contrafácticos, la relación de similitud entre mundos o la función de selección, deben satisfacer ciertos requisitos semánticos. Pero dichos requisitos son insuficientes para darle valores de verdad a los contrafácticos. Esto quiere decir que, en la concepción clásica, la semántica no le otorga valores de verdad a los contrafácticos, independientemente de un contexto de uso. Si el objetor prefiere evitar hablar de "semántica" sin valores de verdad puede llamarle "semántica" a la teoría general que incorpora el contexto de uso, las proferencias y las presuposiciones de los hablantes, además de la función de selección (o la relación de similitud). Hay apasionantes y difíciles discusiones acerca de cuál es la línea que separa la semántica de la pragmática. Pero esta no es una de ellas. Se

Principia 18(1): 135-151 (2014). 
trata, en realidad de una discusión de palabras.

${ }^{19}$ De hecho, me inclino ligeramente por la primera opción.

${ }^{20}$ El pasaje que cité antes (al final de la sección 2) comienza diciendo "Even if, against my better judgment, the third type of account of true/true subjunctives is correct, I will be ..." (2004, p.31) Este pasaje, leído en su totalidad, sugiere que, si bien DeRose es consciente de las dificultades semánticas ya mencionadas, en último término, apostaría por la extrañeza pragmática para desacreditar el análisis. Considero que esta posible manera de interpretar su crítica es innecesariamente débil.

${ }^{21}$ La sección 6 del artículo de DeRose se ocupa de discutir algunas paráfrasis intuitivas alternativas del condicional de seguridad. Al parecer, estas maneras alternativas de interpretar el condicional no generarían la rareza pragmática mencionada. Los candidatos de Sosa son "(S2) $S$ would believe that $p$ only if it were so that $p$ (S3) $S$ would not believe that $p$ without it being the case that $p$ (S4) As a matter of fact, though perhaps not as a matter of strict necessity, not easily would $S$ believe that $p$ without it being the case that $p$. " (DeRose (2004), p.31) Como a DeRose, a mí me parece que estas lecturas del condicional de fuerza son un tanto forzadas, e innecesariamente complejas. De hecho, uno podría levantar la sospecha de que la forma lógica de S2 es, en realidad, "no es posible (psicológicamente) que $p$ y no $q$ ". "Yo sería feliz sólo si fuera el caso que estoy casado" se parece, después de todo, a "no es posible (psicológicamente) que yo esté casado y que no sea feliz". Y quizás se podría decir algo similar acerca de S3. Sin embargo, no son estos los problemas centrales del análisis de Sosa. Estos pasajes de DeRose ocultan la verdadera dialéctica involucrada en la discusión. Y tienen la desafortunada consecuencia de invitar una respuesta de Sosa que no atiende al problema central.

${ }^{22}$ Esto es, ni (1) ni (2).

${ }^{23}$ El trabajo de Walters (2013) fundamenta nuestra sospecha de que dicha tarea no resultará exitosa.

Principia 18(1): 135-151 (2014). 